\title{
Proceedings of the 16th European powder diffraction conference
}

Powder Diffraction once again hosts a selection of papers presented at a European Powder Diffraction Conference, EPDIC16, held in Edinburgh, Scotland, at the John McIntyre Conference Center of the University of Edinburgh, on 1-4 July 2018.

EPDIC conferences are now steadily playing a role of reference for the community of experts and users of powder diffraction. Led by a stable scientific committee (details of the EPDIC organization can be found at http://epdic.ing. unitn.it/), EPDIC presents the main topics of powder diffraction with emphasis that from edition to edition moves on the themes of the host sites, but above all on emerging topics, with particular attention to applications to science and technology.

As in recent EPDICs, a growing interest has manifested itself around the themes concerning new sources and experimental methods, with sessions dedicated to "New Sources and Instruments for Powder Diffraction", "New developments in instrumentation for sample environments", "XRD diffraction imaging and combined methods", and "Balancing conventional powder diffraction structural approaches with computation and electron diffraction".

Among the traditional themes of the EPDIC, which above all remains a conference on analytical methodologies, the evergreen on "Methods in structure solution and refinement", "Total Scattering and Disorder", and "Microstructure phenomena in thin films".

Special emphasis on "Materials under extreme conditions", and in several branches of materials science and technology ranging from "Nanomaterials: Structural, Microstructural and Surface Aspects" to "Pharmaceutical and biological materials", with dedicated sessions on "Emerging functional materials" and "Energy Materials".

The scientific program was enhanced by the presentations of Bill David (ISIS, Oxford University), winner of the Distinguished Powder Diffractionist award, and Stef Smeets (Stockholm University), winner of the EPDIC Young Scientist Award, plenary talks given by Robert Feidenhans'l (FEEL, DE), Emil Bozin (Oak Ridge, USA), Maria Teresa Fernandez-Diaz (ILL, France), Beatriz Noheda (Groningen,

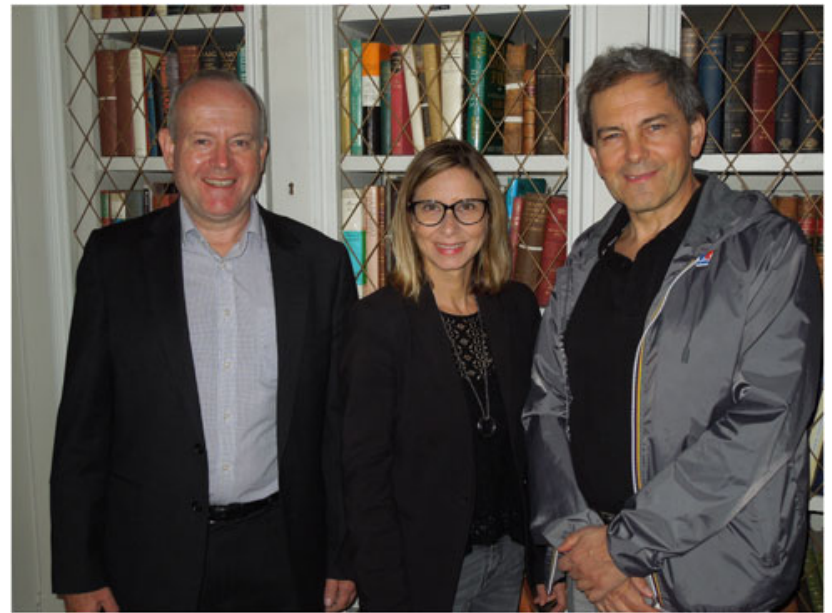

Photo 1: (Colour online) From left to right: Guest Editors Robert Cernik, Michela Brunelli, and Paolo Scardi

NL), Jan Skov Pederson (Aarhus, Denmark), and Gwen Rousse (UPMC, Paris, France), and by the Lachlan's Software Fayre, dedicated to the memory of L. Cranswick.

Further interest around the event was provided by two satellite events, the traditional ICDD half-day workshop and a new two-day edition of the TOPAS user meeting, both held in days preceding the EPDIC.

Pictured from the event (Photo 1) are Guest Editors Robert Cernik, Michela Brunelli, and Paolo Scardi.

The guest editors of this special issue of Powder Diffraction:

Michela Brunelli DUBBLE/ESRF, Grenoble, France

Bob Cernik University of Manchester, Manchester, U.K.

Paolo Scardi University of Trento, Trento, Italy 